\title{
Determination of mass-dependent variations in tungsten stable isotope compositions of geological reference materials by double- spike and MC-ICPMS
}

Kathrin Abraham ${ }^{a}$, Jane Barling ${ }^{a}$, Christopher Siebert ${ }^{b}$, Nick Belshaw ${ }^{a}$, Louise Gall ${ }^{\text {, }}$, and Alex N. Halliday ${ }^{a}$

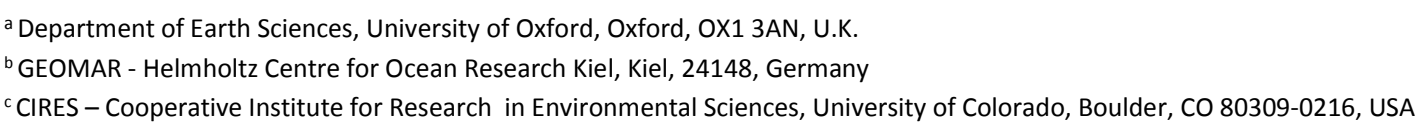

We present a double-spike technique for precise determination of mass-dependent fractionation of tungsten (W) stable isotopes. Instrumental mass bias effects and isotope fractionation during $\mathrm{W}$ separation are corrected for by means of a ${ }^{180} \mathrm{~W}-{ }^{183} \mathrm{~W}$ double-spike added prior to sample dissolution. The separation of $W$ from matrix is achieved via threestep anion-exchange chromatography. Tungsten stable isotopic compositions were determined by multi-collector inductively coupled plasma mass spectrometry (MC-ICPMS). Results are expressed relative to the NIST SRM $3136 \mathrm{~W}$ standard as the permil difference in ${ }^{186} \mathrm{~W} /{ }^{184} \mathrm{~W}\left(\delta^{186} \mathrm{~W}\right)$. The external reproducibility of the NIST SRM $3163 \mathrm{~W}$ standard is \pm 0.05 $\%$ with an average composition of $-0.01 \%$ on ${ }^{186} \mathrm{~W} /{ }^{184} \mathrm{~W}$ ( 2 s.d., $\mathrm{n}=171$ ). Tungsten stable isotope compositions for USGS standard reference materials are as follows: AGV-2 (andesite; $\delta^{186} \mathrm{~W}=0.18 \pm 0.05 \%$, 2s.d., $n=6$ ), SDC-1 (mica schist; $\delta^{186} \mathrm{~W}=0.36 \pm 0.05 \%$, 2s.d., $\mathrm{n}=6$ ), SDO-1 (shale; $\delta^{186} \mathrm{~W}=0.26 \pm 0.06 \%, 2 \mathrm{~s} . \mathrm{d}$., $\mathrm{n}=6$ ), and manganese nodules NOD-A-1 $\left(\delta^{186} \mathrm{~W}=0.07 \pm 0.05 \%\right.$, 2s.d., $\left.n=10\right)$ and NOD-P-1 ( $\delta^{186} \mathrm{~W}=0.31 \pm 0.04 \%$, 2s.d., $\left.n=7\right)$. The longterm external reproducibility ( 2 s.d.) for these rock standards is $\pm 0.05 \%$.

\section{Introduction}

Tungsten $(Z=74)$ is an important component in modern materials due to its very high melting $\left(3422^{\circ} \mathrm{C}\right) /$ boiling point $\left(5930^{\circ} \mathrm{C}\right)$. It has the greatest tensile strength at elevated temperature, and a high density of $19.1 \mathrm{~g} \mathrm{~cm}^{-3}$. These properties render it very suitable for a wide variety of industrial and military applications. ${ }^{1}$ Tungsten is also of great importance in geochemistry. It is highly refractory during condensation, ${ }^{2}$ and thus of great interest in studying early Solar System processes. While the Sun and chondrites ${ }^{3}$ have approximately constant inventory of $W$ when compared to other highly refractory elements (e.g. titanium and hafnium), the majority of $W$ in a terrestrial planet is in its core, the relative amount depending on how oxidising the conditions of metal segregation were. In the case of Earth this is $>90 \%{ }^{4}$ in the case of Mars it is much less. ${ }^{5}$

Tungsten has five stable isotopes: ${ }^{180} \mathrm{~W},{ }^{182} \mathrm{~W},{ }^{183} \mathrm{~W},{ }^{184} \mathrm{~W}$ and ${ }^{186} \mathrm{~W}$, with respective natural abundances of $0.12 \%, 26.50 \%, 14.31 \%, 30.64 \%$ and $28.43 \%{ }^{6}$ To date, $W$ isotope geochemistry has mainly focused on chronometry. Radiogenic ${ }^{182} \mathrm{~W}$, formed by decay of 
${ }^{182} \mathrm{Hf}$, has been used to study processes during the first $\sim 100 \mathrm{Ma}$ of Solar System history. ${ }^{7,8}$ Hafnium and $W$ are fractionated from each other during core formation, leading to divergent $\mathrm{W}$ isotopic compositions between the core and the mantle, which are a function of time. ${ }^{9}$ More recent publications have documented evidence of variations in the radiogenic ${ }^{182} \mathrm{~W}$ signature of the Earth's mantle ${ }^{10,11}$ and given very precise chronologies of asteroidal core formation. ${ }^{12}$

During mantle melting, $\mathrm{W}$ behaves as a highly incompatible element with a bulk distribution coefficient similar to that of Ba. ${ }^{4,13}$ Consequently, within the Bulk Silicate Earth (BSE) (Earth minus core) approximately half of the $\mathrm{W}$ resides in the continental crust. The average $\mathrm{W}$ content of the continental crust is estimated to be $1,000 \mathrm{ppb}$, with a relatively enriched upper continental crust (1,900 ppb) and a more depleted lower and middle crust (600 ppb). 14 The concentration of $\mathrm{W}$ in the ocean is low and constant at $\sim 50 \mathrm{pmol} / \mathrm{L}$ showing conservative behavior. ${ }^{15,16}$ Tungsten has a relatively long residence time in seawater $\left(\sim 61,000\right.$ years $\left.{ }^{15}\right)$ and is present mainly as the tungstate ion $\left(\mathrm{WO}_{4}{ }^{2-}\right)$. Tungsten exhibits oxidation states from -2 to +6 , but the most common oxidation state is +6 . Under oxidising conditions it forms yellow tungsten trioxide, $\mathrm{WO}_{3}{ }^{17}$.

Here we present new techniques for the precise measurement of mass-dependent $W$ stable isotope compositions in geological materials. A wide range of such novel, non-traditional stable isotope systems have been recently developed following the advent of multiplecollector inductively coupled plasma mass spectrometry (MC-ICPMS). Although degrees of mass dependent fractionation are predicted to be small in heavy elements, due to the fact that $\mathrm{W}$ occurs in various oxidation states in nature it has the potential to exhibit significant stable isotope variations in earth materials. Other heavy elements like thallium, ${ }^{18}$ uranium $^{19}$ or barium ${ }^{20}$ display significant low temperature isotopic fractionations. Tungsten has the potential to be a powerful tracer of a variety of geochemical and cosmochemical processes, including variations in core formation of the Earth and other planetary bodies, magma formation in different geological settings and diverse fluid-mediated processes in the continents and oceans.

Few data on tungsten stable isotope fractionation have been published so far. Irisawa and Hirata $^{21}$ were the first to study the mass-dependent fractionation of $W$ isotopes in sediments and igneous rocks. They used Re to monitor instrumental mass bias during analysis on a MC-ICPMS, applying a modified exponential law. In a survey of geological materials ${ }^{22}$ they found an overall degree of isotopic variation of $0.21 \%$ omu $\mathrm{am}^{-1}(+-0.05 \%$ in standard solutions and $0.1 \%$ in samples) with $\mathrm{W}$ isotopic ratios from hot spring deposits being heavier than the of standard reference material NIST SRM 3163. Irisawa et al. ${ }^{23}$ reported the first stable isotope ratios among chondritic materials for both, ordinary and carbonaceous chondrites. Breton and Quitté ${ }^{24}$ developed an improved chemical separation procedure showing quantitative recovery of $W$, which is essential to avoid mass fractionation during chemical treatment, when analysing unspiked samples via sample- 
standard bracketing or external normalisation techniques using MC-ICPMS. They used Hf to monitor instrumental mass bias effects and determined $W$ isotope ratios for a range of igneous rocks, chondrites and iron meteorites with stable isotope compositions ranging from -0.05 to $+0.36 \%$ o per amu. Most recently, Krabbe et al. ${ }^{25}$ reported stable $W$ isotope measurements, using a double-spike method. Their initial results show a much smaller magnitude of $\mathrm{W}$ isotope fractionation in meteorites than those of Breton and Quitté ${ }^{24}$.

Our double-isotope spike method for measuring mass-dependent fractionation of W stable isotopes with MC-ICPMS to high precision is combined with an optimized chemical separation technique. This combination minimises potential interferences and allows correction of possible mass dependent fractionation during chemical separation procedures as well as mass spectrometry. This in turn makes the analysis of a wide variety of sampletypes possible.

\section{Materials and Methods}

\subsection{Sample dissolution}

Samples were prepared in the clean room facilities of the department of Earth Sciences, Oxford University, UK. Depending on W concentration, between 5 and $300 \mathrm{mg}$ of sample material were spiked with a ${ }^{180} \mathrm{~W}-{ }^{183} \mathrm{~W}$ double-spike (see 3.3). Samples were then dissolved in a mixture of $\mathrm{HF}-\mathrm{HNO}_{3}-\mathrm{H}_{2} \mathrm{O}_{2}$ (5:4:1) in pre-cleaned Savillex vials. Tungsten may form volatile $\mathrm{W}$-flouride complexes during sample digestion. ${ }^{26}$ It is therefore essential to avoid loss of $\mathrm{W}$ before the spike-sample equilibration as this would lead to inaccurate $\mathrm{W}$ isotope compositions. For this reason digestion temperatures are kept low. The first dissolution step starts at room temperature for several days and then moves to the hotplate for two days at $90^{\circ} \mathrm{C}$. The samples are subsequently evaporated to dryness at $90^{\circ} \mathrm{C}$. The samples were then re-dissolved in a $\mathrm{HNO}_{3}-\mathrm{H}_{2} \mathrm{O}_{2}$ (4:1) mixture to remove organic compounds, which have the potential to form molecular interferences on ${ }^{183} \mathrm{~W}$ during measurements (e.g. $\mathrm{C}_{13} \mathrm{H}_{27}{ }^{+}$, $\mathrm{C}_{14} \mathrm{H}_{15}{ }^{+}$, or $\left.\mathrm{C}_{15} \mathrm{H}_{3}{ }^{+}\right)^{22}$ and to remove Os (isobaric interference on ${ }^{186} \mathrm{~W}$ ). After drying, the samples were first dissolved in a mixture of $6 \mathrm{MHCl}-1 \mathrm{MHF}$, evaporated to dryness again and then taken up in $1 \mathrm{M} \mathrm{HF}$ to be diluted to $0.1 \mathrm{MHF}$ for loading on the anion exchange column. Small amounts of insoluble fluorides $\left(\mathrm{MgF}_{2}, \mathrm{CaF}_{2}\right.$, or $\left.\mathrm{AlF}_{3}\right)$ were removed via centrifuging (4000 G, 5min). No $W$ should be present in the flourides, however the double-spike technique accounts for any loss of $W$ at this stage (i.e. after spike-sample equilibration).

\subsection{Chemical separation}

Tungsten was separated from the sample matrix using a three-stage anion exchange chromatography (BIORAD AG 1X8, 200-400 mesh) modified and expanded from Sahoo et al. ${ }^{27}$ The modifications include longer washouts, repetition of the first anion exchange separation and the addition of a third column designed to completely remove Mo and Fe from $\mathrm{W}$. In addition, several unwanted elements $(\mathrm{Mg}, \mathrm{Cr}, \mathrm{Mn}, \mathrm{Ni}, \mathrm{Cu}$ and $\mathrm{Zr}$ ) are washed out 
in the first $5 \mathrm{ml}$ of $5 \mathrm{M} \mathrm{HCl}$ after the acid change during this last step. An outline of the separation procedure is given in Table 1 . The final $W$ cut is collected using $35 \mathrm{ml} 5 \mathrm{M} \mathrm{HCl}$, which is evaporated to dryness and oxidized three times in a small volume of concentrated $\mathrm{HNO}_{3}$ and $30 \% \mathrm{H}_{2} \mathrm{O}_{2}(4: 1)$ to break down any organic material added to the solution from the resin. Finally, the sample was dissolved in $0.2 \mathrm{M} \mathrm{HNO}_{3}$ and $0.1 \mathrm{M} \mathrm{HF}$ and diluted to 100 ppb for analysis. The yield through chemistry was typically $50-80 \%$. Total procedural blanks varied between 100 and $500 \mathrm{pg} \mathrm{W}$ rendering blank corrections unnecessary given that about 100-200 ng W was analysed for each sample (the maximum observed blank contribution of $0.5 \%$ would cause variation within the error even if the blank would have a significantly larger isotope fractionation than the sample).

\section{Mass spectrometry}

\subsection{MC-ICPMS procedure}

Tungsten isotopic compositions were analysed on a $\mathrm{Nu}$ Instruments (Wrexham, UK) $\mathrm{Nu}$ Plasma-HR double focusing multi-collector ICPMS at the Department of Earth Sciences, Oxford University. Samples were run in $0.2 \mathrm{M} \mathrm{HNO}_{3}$ and $0.1 \mathrm{M} \mathrm{HF}$ at a concentration of $\sim 100$ $\mathrm{ppb}$ and introduced to the plasma via a microconcentric PFA nebulizer (ESI Scientific; $50 \mu \mathrm{l}$ $\left.\mathrm{min}^{-1}\right)$. In order to enhance sensitivity and minimise oxide formation, a desolvating nebulizer system (Nu Instruments, DSN-100) was used. Between analyses the desolvating system was washed by aspirating a solution of $1 \mathrm{M} \mathrm{HNO}_{3}$ and $0.5 \mathrm{M} \mathrm{HF}$ for 5 min and then re-equilibrated with $0.2 \mathrm{M} \mathrm{HNO}_{3}$ and $0.1 \mathrm{M} \mathrm{HF}$ running solution for $100 \mathrm{sec}$. The sample was always washed out to a signal level below the baseline before starting the next measurement. Further details of the instrument running conditions are shown in Table 2. The ion beams for all $W$ isotopes and for the monitoring of important potential isobaric interferences ${ }^{180} \mathrm{Hf},{ }^{180} \mathrm{Ta}$, ${ }^{186}$ Os) were measured simultaneously in static collection mode on an array (Table 3 ) of Faraday detectors equipped with $10^{11} \Omega$ resistors. All measurements were performed relative to the $W$ standard reference material SRM 3163 from the National Institute of Standards and Technology (NIST). Deconvolution of the raw double spike data followed the geometrical calculations of Siebert et al. ${ }^{28}$ with data reported in the delta $(\delta)$ notation as permil (\%o) deviations in ${ }^{186} \mathrm{~W} /{ }^{184} \mathrm{~W}$ relative to this NIST SRM 3163:

$\delta^{186} W=\left[\frac{\left({ }^{186} W /{ }^{184} W\right)_{\text {sample }}}{\left({ }^{186} W /{ }^{184} W\right)_{\text {SRM } 3163}}-1\right] * 1000$

\subsection{Double-spike design and preparation}

The standard double-spike technique requires that the element to be analysed has at least 4 naturally occurring isotopes, two isotopes that are spiked and two for a natural ratio. 
Tungsten has 5 naturally occurring stable isotopes. The double-spike technique has several advantages over sample-standard bracketing and external normalisation using another element. By spiking at the beginning of sample processing, isotope fractionation during measurement and chemical separation can be corrected for, which means that a quantitative yield through chemical separation, though desirable, is not required for reliable data. Matrix effects resulting from differences between samples and pure standards are minimized because instrumental mass bias is monitored and corrected for using a spiked $\mathrm{W}$ isotope ratio rather than by the use of an external element that may not behave similarly to $W$ in the instrument or by relying on a comparison to a matrix free standard. Optimal double-spike composition and the optimal spike-sample ratios have been calculated by Rudge et al. $^{29}$ using algebraic inversion to generate the lowest possible error in the measurement of the natural fractionation factor between the sample and the standard $(\alpha)$. The mathematical iteration and the error curves for these calculations were created using the MATLAB protocol provided by Rudge et al. ${ }^{29}$ For detailed discussions of the double-spike techniques see for example Johnson and Beard, ${ }^{30}$ Galer, ${ }^{31}$ Siebert et al. ${ }^{28}$ Albarède $^{32}$.

According to these theoretical calculations, the combination of ${ }^{180} \mathrm{~W}$ and ${ }^{183} \mathrm{~W}$ results in the lowest theoretical errors on a given isotope fractionation and the least sensitivity of these errors to different double-spike/standard proportions. ${ }^{180} \mathrm{~W}$ and ${ }^{183} \mathrm{~W}$ are the two least abundant $W$ isotopes and although it is preferable to use low abundance isotopes as spike isotopes, the natural abundance of ${ }^{180} \mathrm{~W}(0.012 \%)$ is so low that difficulties can arise with the precise and accurate determination of this isotope in the reference material (NIST SRM 3163) during spike and standard calibrations, this will be further discussed below. In addition, ${ }^{180} \mathrm{~W}$ is sensitive to isobaric interferences from ${ }^{180} \mathrm{Hf}(35.08 \%)$ and $\mathrm{Ta}(0.012 \%)$.

Metal powders of ${ }^{180} \mathrm{~W}$ (91.4\% enriched) and ${ }^{183} \mathrm{~W}$ (99.8\% enriched) were purchased from ISOFLEX (San Francisco, CA, USA). Metal powder was preferred over oxide powder, as the oxide powder is difficult to dissolve. Both powders were weighed separately in pre-cleaned and pre-weighed FEP-bottles and dissolved overnight using $15 \% \mathrm{H}_{2} \mathrm{O}_{2}$ (Romil UpA, Ultra Purity Reagent) and afterwards acidified with $0.2 \mathrm{M} \mathrm{HNO}_{3}$ and $0.1 \mathrm{M} \mathrm{HF}$. The single dissolved spikes were then mixed together gravimetrically to make the double-spike solution. The calculations of Rudge et al. ${ }^{29}$ indicate that two different combinations of natural isotopes with different proportions of the spike isotopes ${ }^{180} \mathrm{~W}$ and ${ }^{183} \mathrm{~W}$ will result in precise isotope measurements. Both combinations were tested here: "mix-high ${ }^{180} \mathrm{~W}$ " $\left({ }^{180} \mathrm{~W}=57.53 \%\right.$ and $\left.{ }^{183} \mathrm{~W}=42.47 \%\right)$ with ${ }^{186} \mathrm{~W} /{ }^{184} \mathrm{~W}$ as the natural isotope ratio and "mix-low ${ }^{180} \mathrm{~W}$ " $\left({ }^{180} \mathrm{~W}=51.02 \%\right.$ and ${ }^{183} \mathrm{~W}=48.98 \%$ ) with ${ }^{186} \mathrm{~W} /{ }^{182} \mathrm{~W}$ as the natural isotope ratio (Table 4). The actual mixing of the spikes resulted in a composition of the "mix-high ${ }^{180} \mathrm{~W}$ " of ${ }^{180} \mathrm{~W}=57.56 \%$ and ${ }^{183} \mathrm{~W}=42.44 \%$ and of "mix-low ${ }^{180} \mathrm{~W}$ " of ${ }^{180} \mathrm{~W}=51.02 \%$ and ${ }^{183} \mathrm{~W}=48.98 \%$, very close to the optimal theoretical compositions calculated by Rudge et al. ${ }^{29}$ The optimal doublespike/standard proportions are 0.3636 and 0.5075 for "mix-high ${ }^{180} \mathrm{~W}$ " and "mix-low ${ }^{180} \mathrm{~W}$ ", respectively. After preparation, both double spikes were diluted to a 10ppm solution with $0.2 \mathrm{MHNO}_{3}$ and $0.1 \mathrm{MHF}$. 


\subsection{Calibration of the double-spike}

The first step in the calibration of each double spike was to determine its isotopic composition in a single session together with the isotopic composition of the NIST SRM 3163 standard. During this session the SRM 3163 and double spike solutions were measured alternately. The collector arrangement used can be seen in Table 3. In order to determine the instrumental mass bias during these measurements, pure standard and spike solutions were doped with Re standard (AA, Lot \# 62-060860, Stock \# 13817) for external normalization. ${ }^{20,28,33}$ The mass bias correction, assumed the natural abundances of ${ }^{185} \mathrm{Re}$ $(0.3740 \%)$ and ${ }^{187} \operatorname{Re}(0.6260 \%)$, as given by $\mathrm{NIST}^{6}$, and used the exponential fractionation law. The assumption that $\mathrm{W}$ and Re show similar fractionation behavior is supported by Irisawa and Hirata ${ }^{22}$, however this assumption is not critical because only pure spike and standard solutions are analyzed and the corrected ratios only have to be defined precisely relative to each other. Matrix effects and possible effects resulting from mass bias correction using Re are negligible provided that all measurements are performed under the same analytical conditions, i.e. during the same measurement session. While the $\mathrm{W}$ isotope ratios determined in this way are not absolute values, the isotope ratios of the double spikes are precisely defined relative to the NIST SRM 3163 standard W ratios. These relative isotopic compositions of the double-spike and the NIST SRM 3163 standard solutions can be found in Table 5.

In the second step of the calibration the robustness of the double-spike was tested by determining the isotopic compositions of a range of volumetrically prepared doublespike/NIST SRM 3163 mixtures and deconvolving the data using the previously determined isotopic compositions for the double spike and NIST SRM 3163. A well calibrated doublespike will result in $\delta^{186} \mathrm{~W}$ values close to zero and with a low error for a large range of different standard/double-spike proportions. The calibration results for "mix-high ${ }^{180} \mathrm{~W}^{\text {" and }}$ "mix-low ${ }^{180}$ W" double spikes are illustrated in Figure 1.

The calculations of Rudge et al., ${ }^{29}$ indicate that the "mix-low ${ }^{180} \mathrm{~W}$ " double-spike should have the lowest errors for a wide range of double-spike/standard ratios (solid line in Figure 1d). The "mix-high ${ }^{180} \mathrm{~W}$ " is theoretically somewhat inferior, as the shape of the calculated error curve is slightly asymmetric, limiting the range of spike/standard ratios with low predicted errors (solid line in Fig. 1b). However, our measurements (black diamonds) show that "mix high ${ }^{180}$ W" results in an overall wider range of possible double-spike/standard ratios. The differences between the calculated and measured results is most likely due to variations in the enrichment factors of non-spike isotopes in the spikes relative to "ideal" clean spike used for the calculations. In addition, the concentration of ${ }^{186} \mathrm{~W}$ in "mix-low ${ }^{180} \mathrm{~W}$ " was too low for accurate analysis ( ${ }^{186} \mathrm{~W}<0.005 \mathrm{~V}$ signal) (Table 4), and the double-spike had to be doped with $0.1 \%$ NIST SRM 3163 in order to increase the signal on ${ }^{186} \mathrm{~W}$ for calibration. Therefore, although both spike solutions result in good reproducibility of the NIST SRM 3163 
standard solution ("mix-high ${ }^{180} \mathrm{~W}$ " $\pm 0.081 \%$, $n=48$; "mix-low ${ }^{180} \mathrm{~W}$ " $0.039 \%, \mathrm{n}=39$ ) albeit with a narrower range of spike sample ratios for "mix-low $180 \mathrm{~W}$ ", "mix-high ${ }^{180} \mathrm{~W}$ " was used as the spike for all subsequent analysis.

During calibration of the double-spike solutions it was observed that the internal analytical errors on the ${ }^{183 / 180} \mathrm{~W}$ ratio were substantially higher than on the ${ }^{182 / 180} \mathrm{~W}$ ratio, even though the abundance of ${ }^{182} \mathrm{~W}$ was a factor of 10 lower than the abundance of ${ }^{183} \mathrm{~W}$. The same effect was not observed in the NIST SRM 3163 standard solution analysed in the same runs. Although noisy ${ }^{183} \mathrm{~W}$ signals have been observed in previous studies $7,11,12,22,24$ and have been mainly attributed to effects of chemical treatment or molecular interferences formed in the plasma, these explanations cannot account for the absence of the observed effect in the SRM 3163. It was found that the ${ }^{183} \mathrm{~W}$ signal noise in the spike solution vanished after the pure spike solution was passed through a chemical purification procedure, using a modified version of that in Quitté et al. ${ }^{34}$. We therefore speculate that an impurity in the $\mathrm{W}$ metal used to produce the spike solutions might have caused interferences on mass 183. Whatever the exact reason, the problem was removed by passing the spike solution through ion chromatography. The unspiked standard solution (NIST SRM 3163) was treated using the same procedure. Subsequently the spike and standard were calibrated as described above.

\section{Results and Discussion}

\subsection{Parameters influencing the quality of measurements}

\subsubsection{Radioactive decay of $\mathrm{Hf}$}

The isotopic abundance of ${ }^{182} \mathrm{~W}$ in geological samples can increase via decay of ${ }^{182} \mathrm{Hf}$ with a relatively short half-life of $8.9 \times 10^{6} \mathrm{yrs}$ which is used as a tracer for processes during the accretion of the Earth. Irisawa and Hirata ${ }^{22}$ observed no significant levels of isotopic variation of ${ }^{182} \mathrm{~W}$ in modern geochemical reference materials (JB-1, JA-3, JCh-1, JMn-1 and HSD), which is consistent with previously reported $W$ isotope data ${ }^{27}$. Small variations of < $20 \mathrm{ppm}$ in the ${ }^{182} \mathrm{~W}$ abundance relative to modern rocks have been reported for rocks predating the "late veneer" event and komatiites possibly derived from a primordial, not homogenized mantle source. ${ }^{10,11}$ However, this observed terrestrial variation in ${ }^{182} \mathrm{~W} /{ }^{184} \mathrm{~W}$ is a factor 2 to 4 lower than the precision achievable here. Furthermore, this study does not include any such very early mantle derived rock samples. Therefore, radiogenic ingrowth on ${ }^{182} \mathrm{~W}$ is negligible for the purposes of this method.

\subsubsection{Interferences and matrix effects during sample analysis}

Three $\mathrm{W}$ nuclides $\left({ }^{180} \mathrm{~W},{ }^{184} \mathrm{~W}{ }^{186} \mathrm{~W}\right)$ have potential isobaric interferences from hafnium (Hf), tantalum ( $\mathrm{Ta}$ ) and osmium (Os) isotopes. In addition, argides, oxides and nitrides containing rare earth elements (REE) can result in polyatomic interferences on tungsten isotopes. An overview of interferences on W isotopes can be found in Breton and Quitté ${ }^{24}$. 
Although it is possible to correct for isobaric interferences and resolve polyatomic interferences from sample $\mathrm{W}$ signals, these procedures should be avoided because they are sources of potential analytical error or reduce the signal strength of the analyte, respectively. Therefore, care was taken to optimize the chemical separation procedure to remove all potential interferences. The double-spike technique allows extra flexibility here because chromatographic yield can be sacrificed for purity. Analysis of analyte solutions of USGS reference materials (AGV-2 and Nod-A-1) by ICPMS (Thermo Scientific, Element $2^{\mathrm{TM}}$ ) has shown that our chemical separation procedure effectively removes all elements that can cause isobaric or polyatomic interferences (e.g. Ti, Fe, $\mathrm{Zr}$, Nb, Mo, REE, Hf, Ta, Os).

In addition, during each isotope measurement $\mathrm{Os}, \mathrm{Hf}$ and $\mathrm{Ta}$ are routinely monitored simultaneously with $\mathrm{W}$ to assure that no isobaric interferences are present.

To test what effect matrix elements with the potential to form polyatomic interferences would have on $\mathrm{W}$ isotope ratio measurements, $\mathrm{Mg}, \mathrm{K}, \mathrm{Mo}$ and $\mathrm{Nb}$ were added to the NIST SRM 3163 standard solution. This test resulted in no measurable effect on the precision and accuracy of the analyzed NIST solutions (Fig 2). However, the presence of Mo suppresses the ionization efficiency and the $\mathrm{W}$ signal decreases by up to a factor of 2 if the Mo/W ratio becomes as high as 4 .

In theory, isobaric interferences can be corrected during measurements by analyzing a noninterfering isotope or if possible isotope ratio of the respective element and using the obtained signal/ratio to subtract the interfering element signal from the tungsten signal after correction for instrumental mass bias. If an isotope ratio of an interfering element can be monitored the instrumental mass bias for this element can be directly determined and corrected for (see Table 3 for collector setup). If only one mass of the interfering element can be analyzed then in most cases the fractionation factor of the element in question, in this case $W$, is used. In both cases an exponential fractionation law would be assumed.

In order to test the correction described above, NIST SRM $3163 \mathrm{~W}$ (W=100 ppb) was spiked and standard solutions of $\mathrm{Hf}, \mathrm{Ta}$ and $\mathrm{Os}$ were added in different concentrations (1ppb to $0.4 \mathrm{ppm})$.

In the case of $\mathrm{Hf}$ and Os, this correction method failed and even small amounts of $\mathrm{Hf}$ and Os present in the analyte could not be corrected for. One of the possible reasons for this failure might be very different behavior of $\mathrm{Os}$ and $\mathrm{Hf}$ in the plasma with respect of $\mathrm{W}$. Tantalum signals of up to $8 \mathrm{~V}$ on ${ }^{181} \mathrm{Ta}$ can be corrected for within the 2 s.d. envelope of the $\mathrm{W}$ instrumental reproducibility.

\subsubsection{Chemical separation procedure}


In order to further investigate the quality of our chemical separation procedure we performed controlled matrix tests. ICP-standard solutions (Alfa Aesar) of geologically abundant matrix elements ( $\mathrm{Mg}, \mathrm{Al}, \mathrm{Ca}, \mathrm{Fe}, \mathrm{Ti}, \mathrm{V}, \mathrm{Mn}, \mathrm{Co}, \mathrm{Ni}, \mathrm{Sr}, \mathrm{Zr}, \mathrm{Mo}, \mathrm{Ce}, \mathrm{Nd}, \mathrm{Sm}, \mathrm{Er}, \mathrm{Hf}, \mathrm{Ta}$, Os and $\mathrm{Pb}$ ) were mixed together in similar proportions as silicate rocks and then added to the spiked W NIST SRM 3163 standard solution. After processing the mixed solution through the separation chemistry, the purified $W$ solutions yielded isotopic compositions identical to the untreated NIST standard reference solution within error (Fig. 3). In addition, we measured the $\delta^{186} \mathrm{~W}$ of a chemically processed, spiked W-solution (NIST SRM 3163) to the same unprocessed solution (Fig. 3). Isotopic ratios of the two solutions were identical within error, demonstrating that potential stable isotope fractionation during chemical separation is corrected for by the double-spike technique (see above).

\subsection{Reproducibility and accuracy of $W$ isotopic measurements}

The long-term instrumental reproducibility of spiked NIST SRM $3163 \mathrm{~W}$ standard is $\delta^{186} \mathrm{~W}=$ $-0.01 \pm 0.05 \%$ o ( 2 s.d., $n=171$ ) (Fig 4), analysed over a period of 9 months. In addition we analysed pure $\mathrm{W}$ powder (Sigma Aldrich, 99.99\% W). The powder was not processed through the chemical separation procedure, but was dissolved, spiked and subsequently analysed over a period of 6 months, with an average value of $\delta^{186} \mathrm{~W}$ (Sigma Aldrich) = $0.07 \pm 0.04 \%$ o (2 s.d., $n=30$ ).

The external reproducibility of USGS reference materials (andesite AGV-2, mica shist SDC-1, shale SDO- 1 , manganese nodule NOD-A-1 and NOD-P-1) with a range of $\delta^{186} \mathrm{~W}=0.07 \%$ to $0.36 \%$ o $( \pm 0.05,2$ s.d.) is presented in Fig. 5 and Table 6 . All of the analysed solutions were free of interfering elements except for small amounts of Ta, which were corrected for. Interestingly even these few samples show significant differences in their $\mathrm{W}$ isotope compositions with a total spread of $0.3 \%$. It can also be seen that the technique is highly reproducible for rock samples (see also Table 6).

\subsection{Comparison with literature data}

Due to the scarcity of published W stable isotope data, the only USGS reference material in our study for which a comparison with the literature is possible is AGV-2, which has also been analysed by Breton and Quitté ${ }^{24}$ and Krabbe et al. ${ }^{25}$ All given isotope values are relative to NIST SRM 3163. Our value of $\delta^{186} \mathrm{~W}=0.18 \pm 0.05 \%$ (2s.d. $n=6$ ) is derived from six separate dissolutions and chemical separations of the rock standard, thus every data point in Fig. 5 represents a measurement of a fully processed sample.

In their extended abstract, Krabbe et al. ${ }^{25}$ did not see any significant fractionation of AGV-2 from the NIST 3163, but need more data to determine realistic sample reproducibility. In contrast, Breton and Quitté ${ }^{24}$ report a value of $\delta^{186} \mathrm{~W}=0.73 \pm 0.08 \%$ (2.s.d.) which is significantly heavier than our findings and those of Krabbe et $a^{25}$, also using the same NIST SRM 3163 W standard solution. Possible explanations for this discrepancy include 
heterogeneity of the rock standard with respect to $\mathrm{W}$ or analytical artifacts either during chemical separation or instrumental mass bias correction. Our data were produced on a $\mathrm{Nu}$ Instruments ${ }^{\mathrm{TM}}$ MC-ICPMS with standard cones for dry plasma. Both Krabbe et al. ${ }^{25}$ and Breton and Quitté24 used a Thermo Neptune MC-ICPMS. Krabbe et al. ${ }^{25}$ do not specify the cone set used, whereas Breton and Quitté ${ }^{24}$ used Jet and $X$ cones. Our technique as well as Krabbe et al. ${ }^{25}$ made use of a double-spike to account for chemical and instrumental mass fractionation. In contrast Breton and Quitté ${ }^{4}$ employed $\mathrm{Hf}$ doping to account for instrumental mass fractionation relying on a full yield during chemical separation of W. Therefore we speculate that either sample heterogeneity exists or that the difference in methods used for mass bias correction is the cause for the observed discrepancies between the studies.

\section{Summary}

The new double-spike technique presented in this paper allows high-precision measurements of mass-dependent stable $\mathrm{W}$ isotope compositions. It yields a robust instrumental fractionation correction and also corrects for isotope fractionation occurring during the chemical separation procedure. The external reproducibility of NIST SRM 3163 standard was $\pm 0.05 \%$ o ( 2 s.d., $n=171$ ). Replicate measurements of USGS reference materials yield a long-term external reproducibility ( 2 s.d.) of $\pm 0.05 \%$. Although it is difficult to establish accuracy with only few published $W$ isotope data, our analyses of rock standards are highly reproducible over long time periods. This method enables the investigation of $W$ isotope variations in a range of samples and has the potential to provide constraints on a wide variety of geological and cosmochemical problems.

\section{Acknowledgements}

We are grateful for support from Alan Hsieh and Phil Holdship on the mass spectrometer. We also thank Fatima Mokadem and Steve Wyatt for keeping the clean lab running; YuHsuan Liang is thanked for advice and David Cook is thanked for helpful comments. The reviewers are thanked for constructive comments. The authors acknowledge the ERC and STFC for funding. The research material supporting this publication can be accessed by contacting Dr. K. Abraham: kathrin.abraham@earth.ox.ac.uk.

\section{Table caption:}

Table 1 Summary of full chemical procedure for separating $W$ from a silicate matrix by anion-exchange chromatography.

Table 2 Instrumental operating parameters during $\mathrm{W}$ isotope analysis on the Nu plasma-HR

Table 3 Collector configurations used to measure simultaneously tungsten stable isotopes and rhenium (top) for correcting for instrumental mass bias during the double-spike 
calibration. The cup configuration for the rock standard analysis (bottom) monitoring Hf, Ta and Os to correct the interferences on the ${ }^{180} \mathrm{~W},{ }^{184} \mathrm{~W}$ and ${ }^{186} \mathrm{~W}$ signal.

Table 4 The five favourable theoretical spike compositions using ${ }^{183} \mathrm{~W}-{ }^{180} \mathrm{~W}$ double-spike, calculated by the MATLAB error minimization program of Rudge et al. ${ }^{29}$

Table 5 Results of the ${ }^{180}$ W-183 W double-spike and W standard (NIST SRM 3163) calibration for both spike compositions "mix-high ${ }^{180} \mathrm{~W}$ " and "mix-low ${ }^{180} \mathrm{~W}$. The star notes the isotope ratio, which has not been used for calculation in the three isotope space.

Table 6 Tungsten stable isotope data for USGS reference materials. Every measurement represents a full procedural duplicate that was analysed at least twice in different analytical sessions. The 2 s.d. of the single $\delta^{186} \mathrm{~W}$-values refer to either the deviation of the replicate analyses or, if not enough sample solution was available, then to the deviation of the spiked NIST standard analyses during the day of measurement. The external $\delta^{186} \mathrm{~W}$ sample reproducibility was determined to typically be $\pm 0.05 \%$ o $(2$ s.d.).

\section{Figure caption:}

Fig. 1 The figures on the left hand side $(a, c)$ show different proportions of the two favourable double-spike compositions, "mix-high ${ }^{180} \mathrm{~W}$ " and "mix-low ${ }^{180} \mathrm{~W}$ " in a doublespike/reference standard mix (NIST SRM 3163). Measured values for W solutions of all spike/standard ratios are within $\pm 0.08 \%$ of the accurate $\delta$-value. The figures on the right hand side $(b, d)$ show their error. The error for "mix-low ${ }^{180} \mathrm{~W}$ " with a double spike/standard ratio of 0.78 plots outside the diagram in $d$. The solid black error curves denote the uncertainty in the natural fractionation factor $\alpha$ and were calculated using the MATLAB error minimization code of Rudge et al. ${ }^{29}$ with the isotopic concentrations given by ISOFLEX. The results indicate that both double-spike compositions can be used over a wide range of spike/sample ratios. The "mix-high ${ }^{180} \mathrm{~W}$ ", even though it is theoretically the less favourable spike composition using the calculations of Rudge et al. ${ }^{29}$ shows a broader range in different spike/standard compositions and errors closer to the theoretical error curve. Therefore "mix-high ${ }^{180} \mathrm{~W}$ " is our recommended double-spike mix.

Fig. 2 Analyses of a spiked $W$ standard solution doped with different elements in varying concentration ratios to $\mathrm{W}$. The error bars are 2 s.e. of the individual measurement. The dotted lines show the 2 s.d. error on all non-doped $W$ standard solution analyses during the measurement session. a) b) Tests for the accuracy of the interference correction of $\mathrm{Hf}, \mathrm{Ta}$ and Os on W. c) d) Tests for the influence of matrix effect on the W isotope composition. e) Signal loss with increasing Mo concentration.

Fig 3 Analysis of the spiked W standard NIST SRM 3163 to test the effect of the chemical separation procedure. Closed symbols represent a mix of different elemental standard solutions (Alfa Aesar) doped into the W standard solution NIST SRM 3163, which has been put through the separation procedure. Open symbols are the NIST SRM 3163 on its own 
treated by column separation. Error bars are 2 s.e. (0.02 to $0.08 \%$ o) of individual measurements. Dotted lines represent \pm 2 s.d. $( \pm 0.05 \%$ of all NIST measurements.

Fig. 4 Long term instrument reproducibility for the NIST SRM $3163 \mathrm{~W}$ standard. Each point represents the analysis of a spiked NIST SRM $3163 \mathrm{~W}$ standard deconvolved using the method of Siebert et al. $(2001)^{28} . \delta^{186} \mathrm{~W}=-0.01 \pm 0.05 \%$ ( 2 s.d., $n=171$ ). Error bars in the figure are 2 s.e. of the individual measurements.

Fig. 5 Long term external sample reproducibility of several USGS reference materials of different chemical compositions. From top to bottom: AGV-2, mica schist SDC-1, shale SDO1 , manganese nodule NOD-A-1 and NOD-P-1). Each sample point represents the replicate analyses of an individually processed sample powder. The 2 s.d of the single $\delta^{186} \mathrm{~W}$-values refer to either the deviation of the replicate analyses or, if not enough sample solution was available, then to the deviation of the spiked NIST standard analyses during the day of measurement. The external $\delta^{186} \mathrm{~W}$ sample reproducibility was determined to be $\pm 0.05 \%$ (2 s.d.) for the rock standards.

\section{References}

1 E. Lassner and W.-D. Schubert, Tungsten: Properties, Chemsitry, Technology of the Element, Alloys and Chemical Compounds. Springer, 1999, pp. 409-411.

2 K. Lodders, Astrophys., 2003, 591, 1220-1247.

3 A. N. Halliday and D.-C. Lee, Geochim. Cosmochim. Ac., 1999, 63, 4157-4179.

4 H. E. Newsom, K. W. W. Sims, P. D. Noll Jr, W. L. Jaeger, S. A. Maehr and T. B. Beserra, Geochim. Cosmochim. Ac., 1996, 60, 1155-1169.

5 H. Wanke and G. Dreibus, Phil. Trans. R. Soc. Lon. A, 1994, 349, 285-293.

6 NIST Atomic Weights and Isotopic Compositions for All Elements, http://physics.nist.gov/cgi-bin/Compositions/stand alone.pl, (accessed April 2012).

7 D.-C. Lee and A. N. Halliday, International Journal of Mass Spectrometry and Ion Processes, 1995, 146-147, 35-46.

8 C. L. Harper Jr and S. B. Jacobsen, Geochim. Cosmochim. Ac., 1996, 60, 1131-1153.

9 T. Kleine, M. Touboul, B. Bourdon, F. Nimmo, K. Mezger, H. Palme, S. B. Jacobsen, Q.Z. Yin and A. N. Halliday, Geochim. Cosmochim. Ac., 2009, 73, 5150-5188.

10 M. Touboul, I. S. Puchtel and R. J. Walker, Science, 2012, 335, 1065-1069.

11 M. Willbold, T. Elliott and S. Moorbath, Nature, 2011, 477, 195-198.

12 T. S. Kruijer, T. Kleine, M. Fischer-Godde and P. Sprung, Nature, 2015, 520, 534-537. 
13 H. Palme and W. Rammensee, Lunar and Planetary Science XII, Huston, 1981.

14 R.L. Rudnick and S. Gao in Treatise on Geochemistry, ed. R.L. Rudnick, Pergamon, Oxford, UK, 2nd edn., 2014, vol. 4, pp. 1-51.

15 Y. Sohrin, K. Isshiki, T. Kuwamoto and E. Nakayama, Mar. Chem., 1987, 22, 95-103.

16 M. L. Firdaus, K. Norisuye, T. Sato, S. Urushihara, Y. Nakagawa and Y. Sohrin, Geochim. Cosmochim. Ac., 2006, 70, A175.

17 F. A. Cotton, G. Wilkinson, C. A. Murillo and M. Bochmann, Advanced Inorganic Chemistry, John Wiley and Sons, Inc., New York, 6th edn, 1999.

18 M. Rehkämper and A. N. Halliday, Geochim. Cosmochim. Ac., 1999, 63, 935-944.

19 C. H. Stirling, M. B. Andersen, E.-K. Potter and A. N. Halliday, Earth Planet. Sci. Lett., $2007,264,208-225$.

20 K. von Allmen, M. E. Böttcher, E. Samankassou, N. F. Nägler, Chem. Geol., 2010, 277, 70-77.

21 K. Irisawa and T. Hirata, Geochim. Cosmochim. Ac., 2006, 70, A279.

22 K. Irisawa and T. Hirata, J. Anal. At. Spectrom., 2006, 21, 1387-1395.

23 K. Irisawa, Q.-Z. Yin and T. Hirata, Geochem. J., 2009, 43, 395-402.

24 T. Breton and G. Quitté, J. Anal. At. Spectrom., 2014, 29, 2284-2293.

25 N. Krabbe, T.S. Kruijer, G. Budde, and T. Kleine, 46th Lunar and Planetary Science Conference, The Woodlands, Texas, 2015.

26 J. C. Holst, M. B. Olsen, C. Paton, K. Nagashima, M. Schiller, D. Wielandt, K. K. Larsen, J. N. Connelly, J. K. Jørgensen, A. N. Krot, Å. Nordlund and M. Bizzarro, Proc. Nat. Acad. Sci, 2013, 110, 8819-8823.

27 Y. V. Sahoo, S. i. Nakai and A. Ali, Analyst, 2006, 131, 434-439.

28 C. Siebert, T. F. Nägler and J. D. Kramers, Geochem. Geophys. Geosyst., 2001, 2, 1032.

29 J. F. Rudge, B. C. Reynolds and B. Bourdon, Chem. Geol., 2009, 265, 420-431.

30 C. M. Johnson and B. L. Beard, International Journal of Mass Spectrometry, 1999, 193, 87-99

31 S. J. G. Galer, Chem. Geol., 1999, 157, 255-274

32 F. Albarede, P. Telouk, J. Blichert-Toft, M. Boyet, A. Agranier and B. Nelson, Geochim. Cosmochim. Ac., 2004, 68, 2725-2744.

33 E.K. Skierszkan, Amini M and D. Weis, Anal. Bioanal. Chem., 2015, 407, 1925-1935. 
34 G. Quitté, J.-L. Birck, F. Capmas and C.J. Allègre, Geost. Newsletter, 2002, 26, 149160.

35 J. Völkening, M. Köppe and K. G. Heumann, Int. J. Mass Spectrom. Ion Processes, $1991,107,361-368$.

a)

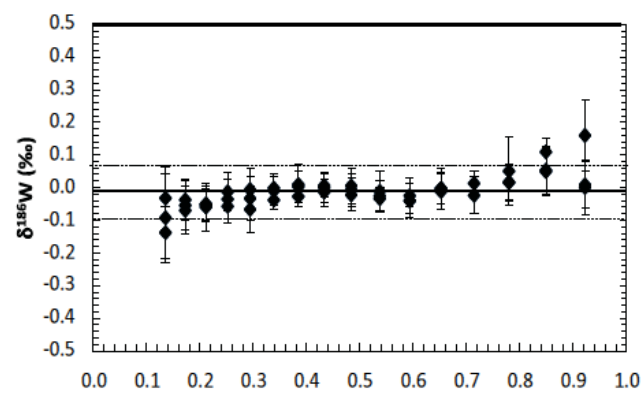

Proportion of "mix-high ${ }^{180} \mathrm{~W}$ " in dbl-spike/standard mix c)

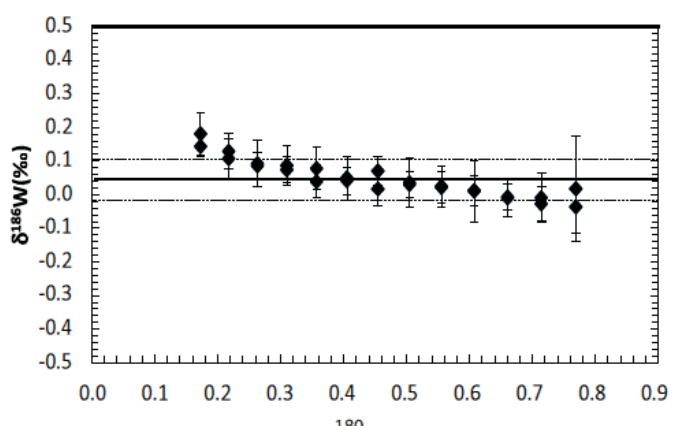

Proportion of "mix-low ${ }^{180} \mathrm{~W}$ " in dbl-spike/standard mix b)

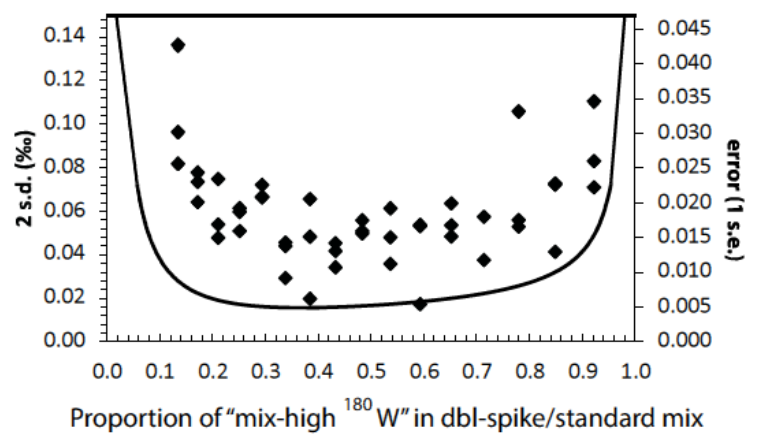

d)

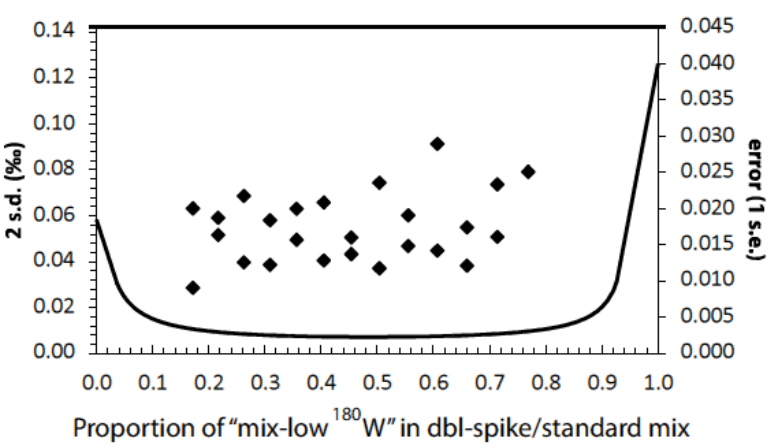



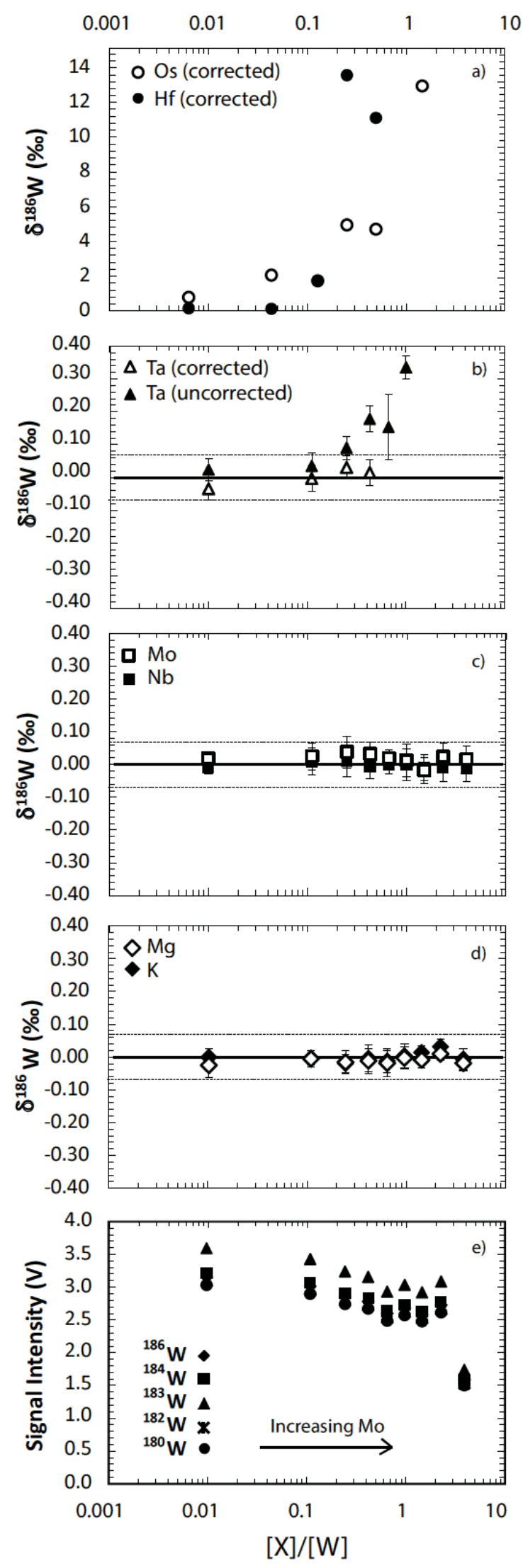

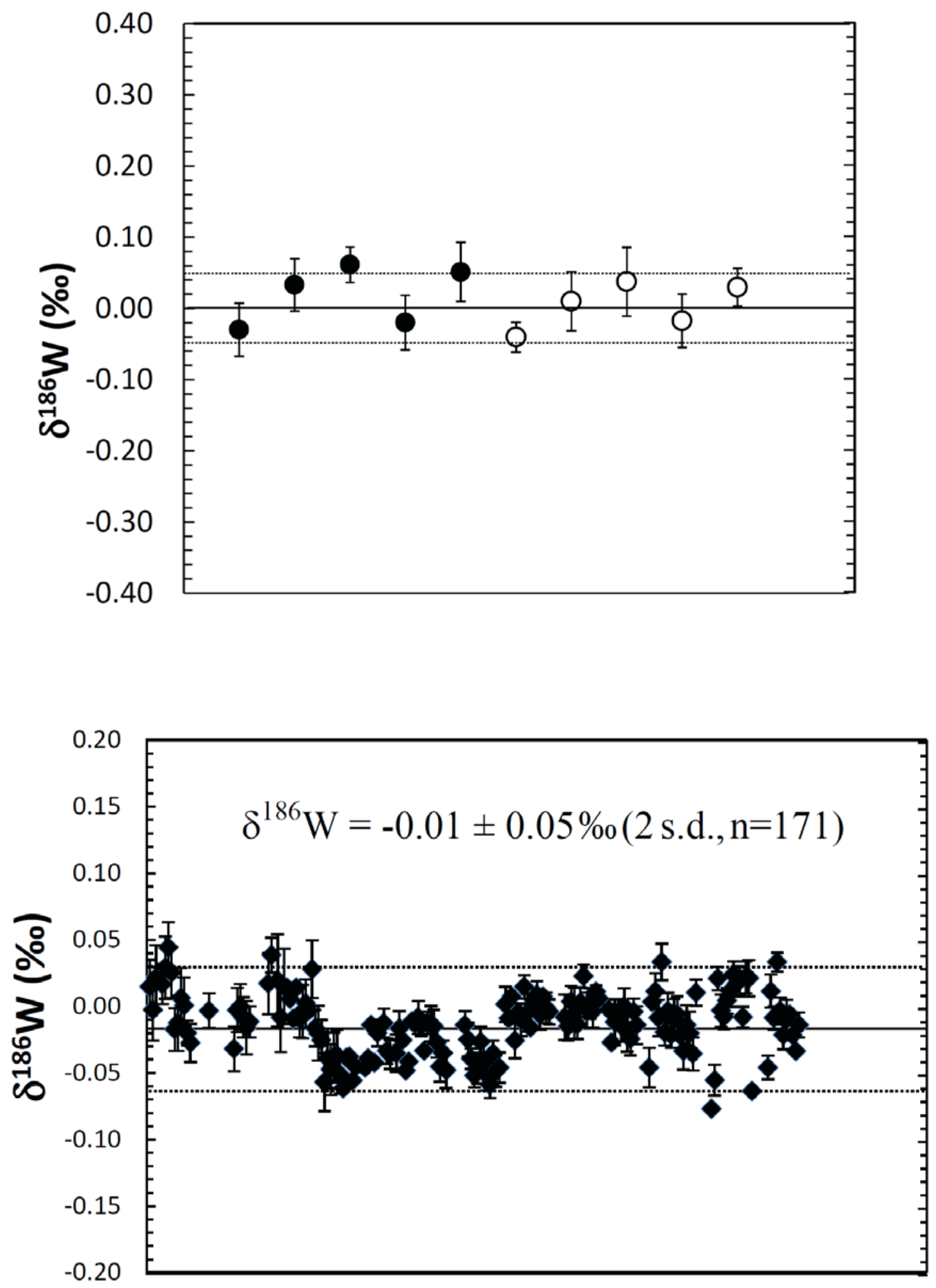

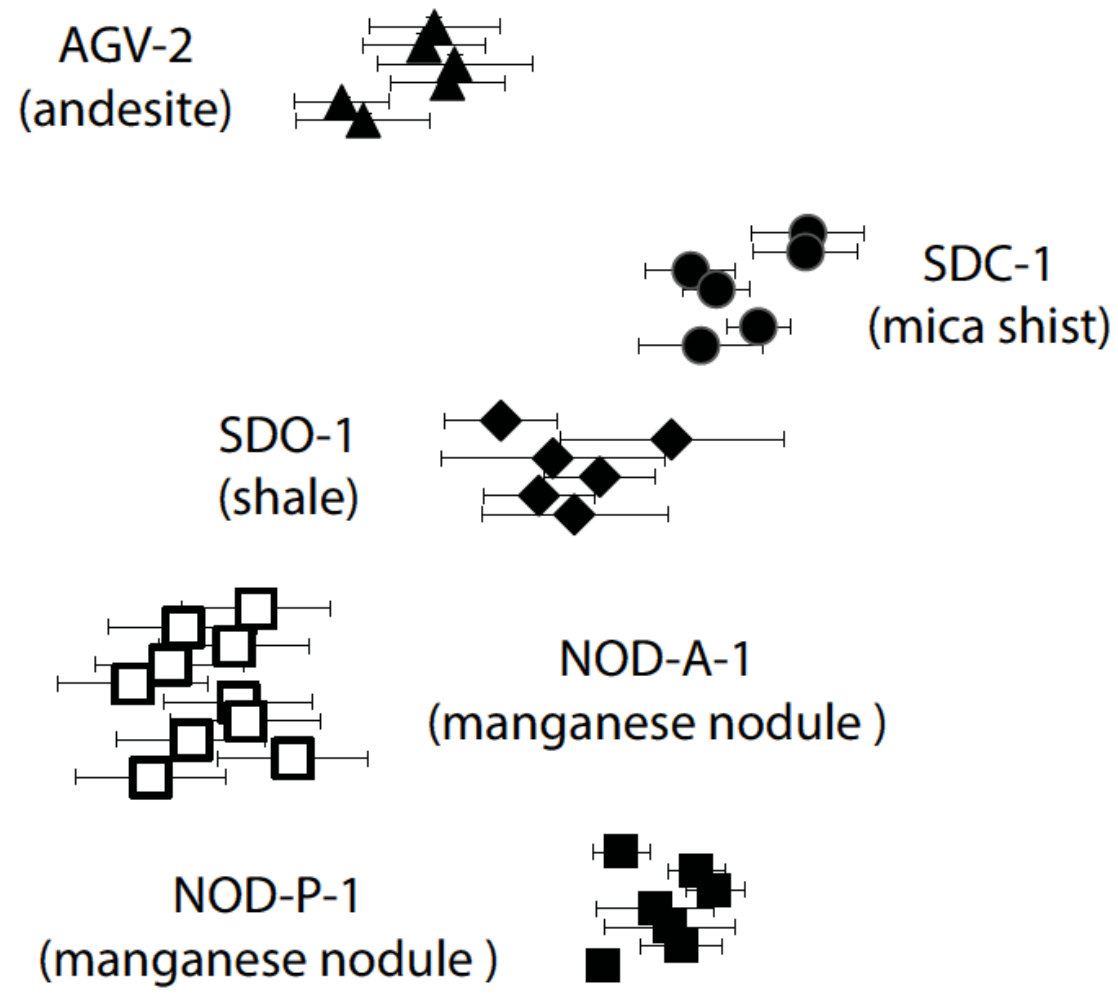

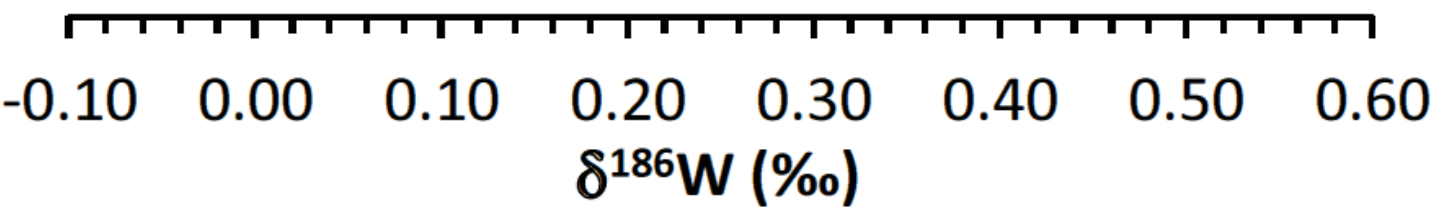




\begin{tabular}{|c|c|c|c|}
\hline Col 1 and Col 2 (AG1-X8; $1.6 \mathrm{ml}$ ) & Acid & Volume (ml) & Elements eluted \\
\hline \multirow[t]{2}{*}{ Precleaning } & $9 \mathrm{M} \mathrm{HCl}+1 \mathrm{M} \mathrm{HF}$ & 8 & \\
\hline & $\mathrm{H}_{2} \mathrm{O}$ (twice) & $8+8$ & \\
\hline Equilibration & $0.1 \mathrm{mM} \mathrm{HF}$ & 8 & \\
\hline Introduction & $0.1 \mathrm{M} \mathrm{HF}$ & 10 & Bulk elements \\
\hline Cleaning step 1 & $0.1 \mathrm{M} \mathrm{HF}+2 \% \mathrm{H}_{2} \mathrm{O}_{2}$ & 4 & $\mathrm{Fe}, \mathrm{Ti}$ \\
\hline Cleaning step 2 & $1 \mathrm{M} \mathrm{HF}+0.2 \% \mathrm{H}_{2} \mathrm{O}_{2}$ & 4 & Minor quantity of $\mathrm{Hf}, \mathrm{Zr}, \mathrm{V}$ residual $\mathrm{Ti}$ \\
\hline Cleaning step 3 & $\mathrm{H}_{2} \mathrm{O}$ & 4 & \\
\hline Cleaning step 4 & $6 \mathrm{M} \mathrm{HCl}$ & $6+4$ & Major Hf, V, Zr, REE, Ta, Sb residual Fe \\
\hline Cleaning step 5 & $8 \mathrm{M} \mathrm{HCl}+0.01 \mathrm{MHF}$ & 4 & $\mathrm{Ag}$ \\
\hline Elution of $\mathrm{W}$ & $6 \mathrm{M} \mathrm{HCl}+1 \mathrm{M} \mathrm{HF}$ & 9 & W \\
\hline Col 3 (AG1-X8; $1.6 \mathrm{ml})$ & Acid & Volume (ml) & Elements eluted \\
\hline \multirow[t]{2}{*}{ Precleaning } & $9 \mathrm{M} \mathrm{HCl}+1 \mathrm{M} \mathrm{HF}$ & 8 & \\
\hline & $\mathrm{H}_{2} \mathrm{O}$ (twice) & $8+8$ & \\
\hline Equilibration & $0.1 \mathrm{M} \mathrm{HF}$ & 8 & \\
\hline Introduction & $0.1 \mathrm{M} \mathrm{HF}$ & 9 & Bulk elements \\
\hline Cleaning step 1 & $\mathrm{H}_{2} \mathrm{O}$ & 4 & \\
\hline Cleaning step 2 & $5 \mathrm{M} \mathrm{HCl}$ & 5 & $\mathrm{Mg}, \mathrm{Cr}, \mathrm{Mn}, \mathrm{Ni}, \mathrm{Cu}, \mathrm{Zr}$ \\
\hline Elution of W & $5 \mathrm{M} \mathrm{HCl}$ & 35 & W \\
\hline
\end{tabular}




\begin{tabular}{|c|c|}
\hline Parameters & Setting \\
\hline RF power & $1300 \mathrm{~W}$ \\
\hline $\begin{array}{l}\text { Acceleration voltage } \\
\text { Resolution mode }\end{array}$ & $\begin{array}{l}6 \mathrm{kV} \\
\text { low resolution } R_{\mathrm{R}}=\mathrm{m} / \Delta \mathrm{m} \sim 400\end{array}$ \\
\hline Sampler cone (Nu instruments) & $\mathrm{Ni}$, B-type $1.0 \mathrm{~mm} \varnothing$ \\
\hline Skimmer cone (Nu instruments) & $\mathrm{Ni}$, WA-type $0.7 \mathrm{~mm} \varnothing$ \\
\hline Scan type & Static mode \\
\hline \multicolumn{2}{|l|}{ Ar gas flow rates $\left(1 \mathrm{~min}^{-1}\right)$} \\
\hline Coolant & 13 \\
\hline Auxiliary & $0.8-1.0$ \\
\hline Uptake rate $\left(\mu \mathrm{L} \min ^{-1}\right)$ & $50-75$ \\
\hline Sample introduction delay & $60 \mathrm{~s}$ \\
\hline Background measurement time & $20 \mathrm{~s}$ \\
\hline Cycle integration time & $10 \mathrm{~s}$ \\
\hline No. Cycles per analysis & 20 \\
\hline Washout time 1 (1M HNO3 - $0.5 \mathrm{M} \mathrm{HF})$ & $300 \mathrm{~s}$ \\
\hline Washout time 2 (0.2M HNO3 - $0.1 \mathrm{M} \mathrm{HF})$ & $100 \mathrm{~s}$ \\
\hline
\end{tabular}




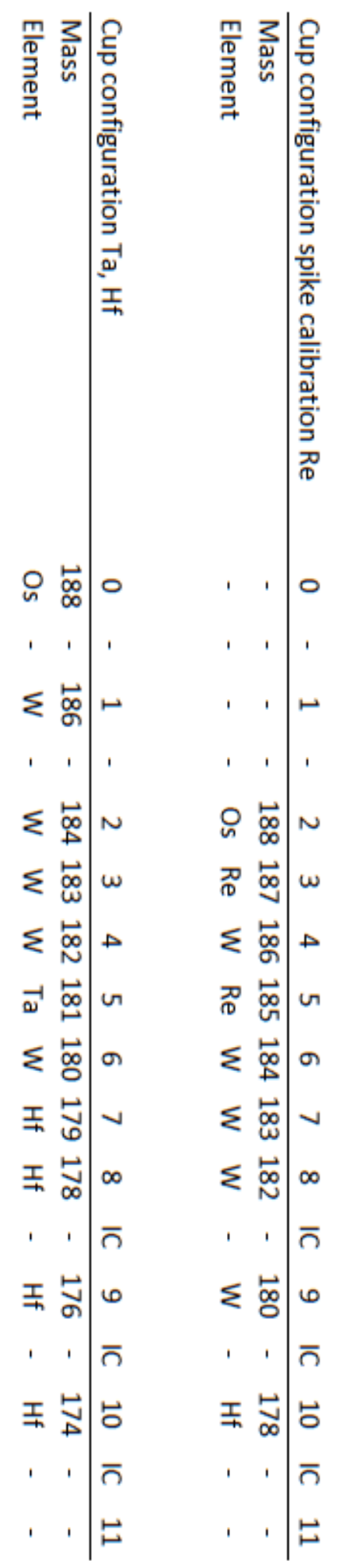




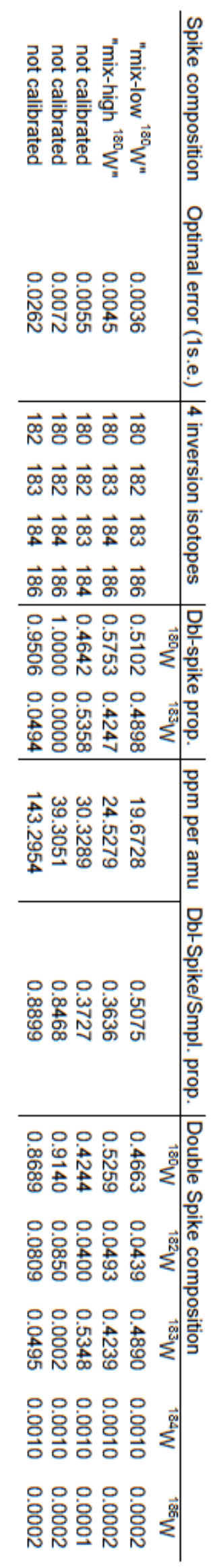




\begin{tabular}{|c|c|c|c|c|}
\hline "mix-high ${ }^{180} \mathrm{~W}$ & ${ }^{186} W /{ }^{184} W$ & ${ }^{183} W /{ }^{184} W$ & ${ }^{182} \mathrm{~W}^{184} \mathrm{~W}^{*}$ & ${ }^{180} \mathrm{~W} /{ }^{184} \mathrm{~W}$ \\
\hline NIST SRM $3163(n=40)$ & 0.9296 & 0.4666 & 0.8630 & 0.00389 \\
\hline 2 s.d. & $4.34 \mathrm{E}-05$ & $2.10 \mathrm{E}-05$ & $5.67 \mathrm{E}-05$ & 2.59E-06 \\
\hline 2.s.d. (ppm) & 47 & 45 & 66 & 666 \\
\hline double-spike $(n=40)$ & 0.073 & 94.9 & 13.26 & 137.8 \\
\hline 2 s.d. & 2.67E-04 & $3.37 \mathrm{E}-02$ & 4.57E-03 & $5.08 \mathrm{E}-02$ \\
\hline 2.s.d. (ppm) & 3654 & 355 & 344 & 368 \\
\hline "mix-low ${ }^{180} \mathrm{~W} "$ & ${ }^{186} \mathrm{~W} /{ }^{182} \mathrm{~W}$ & ${ }^{184} W^{182} W^{*}$ & ${ }^{183} W /{ }^{182} W$ & ${ }^{180} \mathrm{~W} /{ }^{182} \mathrm{~W}$ \\
\hline NIST SRM $3163(n=40)$ & 1.0774 & 1.1588 & 0.5407 & 0.00455 \\
\hline 2 s.d. & $9.57 E-05$ & $5.54 \mathrm{E}-05$ & $1.88 \mathrm{E}-05$ & $2.29 \mathrm{E}-06$ \\
\hline 2.s.d. (ppm) & 89 & 48 & 35 & 502 \\
\hline double-spike $(n=40)$ & 0.0142 & 0.0943 & 8.242 & 10.100 \\
\hline 2 s.d. & $3.61 \mathrm{E}-05$ & $4.38 \mathrm{E}-05$ & $4.75 \mathrm{E}-04$ & $7.20 \mathrm{E}-04$ \\
\hline 2.s.d. (ppm) & 2548 & 464 & 58 & 71 \\
\hline
\end{tabular}

I The concentrations of standard solutions were chosen to be higher than doublespike solutions (NIST SRM $3163=150 \mathrm{ppb}$; double-spike $=125 \mathrm{ppb}$ ), because precise measurements were limited by the low relative natural abundance of ${ }^{180} \mathrm{~W}$ is $0.12 \%{ }^{34}$ and by the high signals of the two double-spike isotopes. 


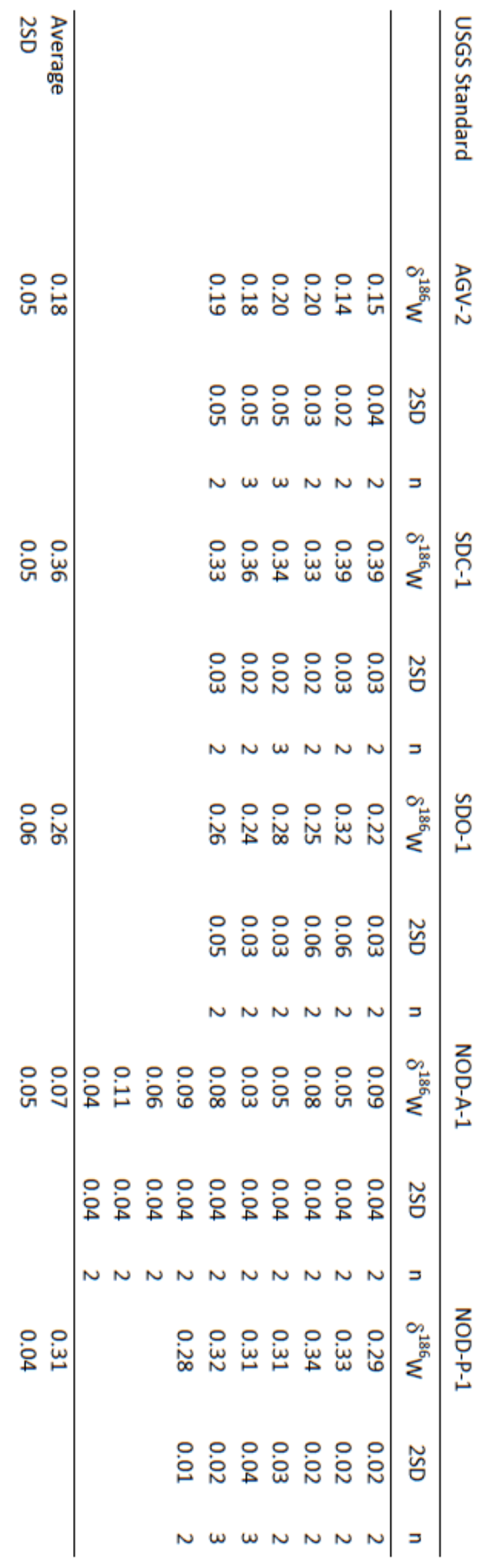

